\title{
A massive reduction of dust particle adhesion in a cyclone by the introduction of a wedge
}

DOI:

$10.1177 / 0954406217728978$

\section{Document Version}

Accepted author manuscript

Link to publication record in Manchester Research Explorer

\section{Citation for published version (APA):}

Zhou, Y., See, T., Zhong, S., Liu, Z., \& Li, L. (2018). A massive reduction of dust particle adhesion in a cyclone by the introduction of a wedge. Institution of Mechanical Engineers. Proceedings. Part C: Journal of Mechanical Engineering Science. https://doi.org/10.1177/0954406217728978

\section{Published in:}

Institution of Mechanical Engineers. Proceedings. Part C: Journal of Mechanical Engineering Science

\section{Citing this paper}

Please note that where the full-text provided on Manchester Research Explorer is the Author Accepted Manuscript or Proof version this may differ from the final Published version. If citing, it is advised that you check and use the publisher's definitive version.

\section{General rights}

Copyright and moral rights for the publications made accessible in the Research Explorer are retained by the authors and/or other copyright owners and it is a condition of accessing publications that users recognise and abide by the legal requirements associated with these rights.

\section{Takedown policy}

If you believe that this document breaches copyright please refer to the University of Manchester's Takedown Procedures [http://man.ac.uk/04Y6Bo] or contact uml.scholarlycommunications@manchester.ac.uk providing relevant details, so we can investigate your claim.

\section{OPEN ACCESS}




\title{
A massive reduction of dust particle adhesion in a cyclone by the introduction of a wedge
}

\author{
Yuanye Zhou ${ }^{1,2}$, Tianlong See ${ }^{2,3}$, Shan Zhong ${ }^{1,2}$, Zhu Liu $^{2,3}$, Lin $\mathrm{Li}^{1,2}$ \\ ${ }^{1}$ School of Mechanical, Aerospace and Civil Engineering, The University of Manchester, Oxford Road, M13 9PL, \\ United Kingdom \\ ${ }^{2}$ Laser Processing Research Centre, The University of Manchester, Oxford Road, M13 9PL, United Kingdom \\ ${ }^{3}$ School of Materials, The University of Manchester, Oxford Road, M13 9PL, United Kingdom \\ *Corresponding author at: School of Mechanical, Aerospace and Civil Engineering, The University of Manchester, \\ Oxford Road, M13 9PL, United Kingdom \\ E-mail address: zhyy2009@163.com
}

\begin{abstract}
ABSTACT
Particle adhesion in a cyclone, such as a cyclonic vacuum cleaner, can significantly reduce its efficiency. An investigation is presented here on the particle adhesion in a cyclone from a vacuum cleaner that consists of a primary separation stage (a
\end{abstract}


cylindrical chamber) and a secondary separation stage (14 cyclones). The flow direction in the primary separation stage was modified by the use of a wedge of 40 $\mathrm{mm} \times 40 \mathrm{~mm} \times 6 \mathrm{~mm}$ at the inlet of the primary separation stage, which affected the particle trajectory in the primary separation stage and the particle inlet position in the cyclone while keeping the air flow direction and velocity (without particles being loaded), the Hamaker constant, particle size, and the particle charge unaffected. The particle inlet position in the cyclone was varied from the lower portion (without wedge) to the upper portion (with wedge). Without the wedge, a spiral pattern of particle (plaster particles, average size $1.13 \mu \mathrm{m}$ ) adhesion onto the inner wall of the cyclone was found and a thicker deposited layer of particles at the cyclone tip region was observed. With the introduction of the wedge, the spiral particle adhesion pattern was not observed and a reduction of particle adhered to the inner wall by up to $94 \%$ was achieved, although there was an increase in the amount of particles entering the cyclone. This demonstrates almost a complete elimination of particle adhesion onto the cyclone wall, without compromising separation efficiencies.

Key words: cyclone, wedge, particle, adhesion, inlet position 


\section{Nomenclature}

$\begin{array}{ll}a & \text { Width of cyclone inlet [m] } \\ b & \text { Height of cyclone inlet [m] } \\ d & \text { Diameter of cyclone tip [m] } \\ D & \text { Diameter of cyclone cylindrical body [m] } \\ D e & \text { Diameter of cyclone vortex finder [m] } \\ H c & \text { Length of cyclone cylindrical body [m] } \\ L & \text { Length of cyclone conical body [m] } \\ S & \text { Length of cyclone vortex finder [m] } \\ t h & \text { Thickness of vortex finder [m] } \\ \alpha & \text { Angle of flow direction [degree] }\end{array}$

Abbreviations

SPs

Spiral Patterns

TAL

Thick Adhesion Layer 


\section{Introduction}

Cyclones are widely used devices in the particle processing technology. A typical cyclone consists of a tangential inlet, a cone shape body, and a vortex finder at the top centre, as shown in Figure 1. Air and mixed particles enter the cyclone through the rectangular tangential inlet. Particles are separated due to the centrifugal force and are collected in the dust collector. Clean air leaves the cyclone through the vortex finder. Basic flow field distribution inside the cyclone is the 'Rankine' vortex structure, which has a solid-body rotation vortex core and a loss-free outer vortex (Hoffmann and Stein, 2002).

It is important to avoid the particle adhesion in the cyclone, as the adhesion would cause the blockage of the cyclone, which deteriorates the performance of the cyclone (O'Callaghan and Cunningham, 2005). Theoretically, the physics of the particle adhesion in the cyclone involves with various forces acting on the particle. In general, the particle adhesion is determined by the capillary force, van der Waals force, electrostatic force and aerodynamic force. The capillary force is mainly determined by the humidity; the van der Waals force is dominated by the Hamaker constant; the electrostatic force is mainly affected by the particle charge; the aerodynamic force are 
determined by the flow velocity and particle size (Mittal and Jaiswal, 2015). In order to reduce particle adhesion, a strong aerodynamic force and weak capillary force and van der Waals force are required. The effect of electrostatic force is determined by the direction of this force. If the direction of electrostatic force is towards to the surface, it aggravates particle adhesion. Otherwise, it helps to reduce particle adhesion.

In addition, particle load rate was found to affect particle adhesion (Houben, 2011). He found that there was a critical particle load rate, which was $2 \mathrm{~g} / \mathrm{kg}$ (mass ratio of particle to air). Weight of particle adhesion increased firstly with increasing particle load rate before it reached $2 \mathrm{~g} / \mathrm{kg}$. After it exceeded $2 \mathrm{~g} / \mathrm{kg}$, the particle adhesion decreased. However, the mechanism behind the effect of particle load rate has not been identified yet, because particle behaviour in cyclone is complex.

In the industry, there are some measures for reducing the blockage of cyclones during operation, such as placing a jet flow tube at the apex of a cyclone (Huang et al., 2013; He et al., 2014), the use of a portable central rod (Mozley, 1979) and the use of vibrating rubber nozzle in a cyclonic vacuum cleaner. However, these methods make the manufacture of the cyclone to be complex which limits their applications. Therefore, there is need to develop an easy way to reduce the particle adhesion in the 
cyclone.

In this study, a novel method to reduce the particle adhesion in a cyclone was reported. In the beginning, we were testing the particle adhesion on some flat sheets placed at the inlet of a vacuum cleaner. It was coincidentally found that the particle adhesion was reduced in the vacuum cleaner. Then, a wedge was designed based on the envelop surface of these flat sheets. Since the interest of this study is mainly on revealing mechanism of particle adhesion reduction behind the wedge, the shape and position of wedge has not been optimized. Instead, the effect of the wedge on the particle adhesion was discussed. Several possible reasons accounting for the particle adhesion reduction were investigated by experimental methods. 


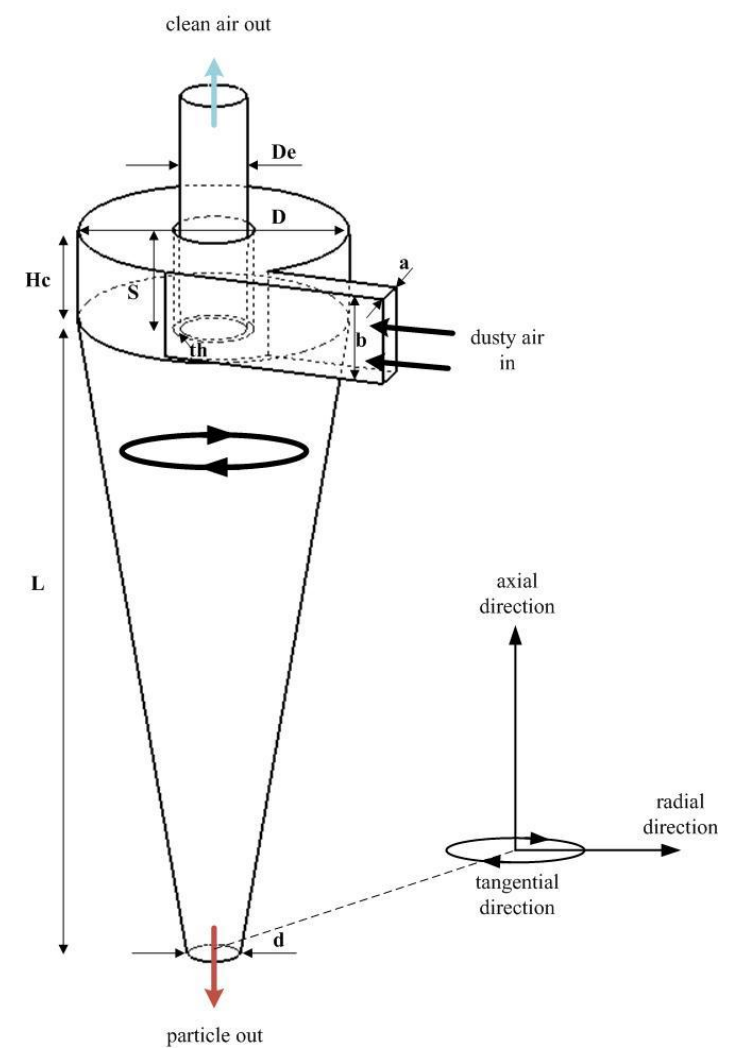

Figure 1 Illustration of a typical cyclone and its key dimensions

\section{Material and Methods}

\subsection{Particles}

As plaster particles can easily form the particle adhesion, Thistle Dura-Finish plaster, manufactured by British Gypsum Ltd. was used in the experiment. The density of this plaster particle is about $2300 \mathrm{~kg} / \mathrm{m}^{3}$. Before the experiment, particles were stored in a 
sealed tank at room temperature, so that particles were not wetted by the humidity.

Figure 2 shows the 2D image of particles under an optical microscope (GXML 3230) and the particle size distribution. Since particles were irregular, the particle size distribution was given in terms of dynamically equivalent diameter, which was measured by a particle sizer (TSI 3321) in a sedimentation tank with diluted particle samples. The average dynamically equivalent diameter of plaster particles was 1.13 $\mu \mathrm{m}$.

a

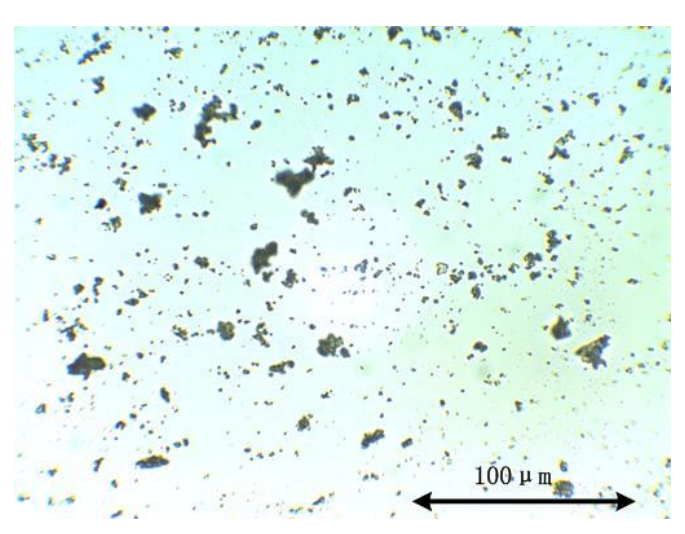

b

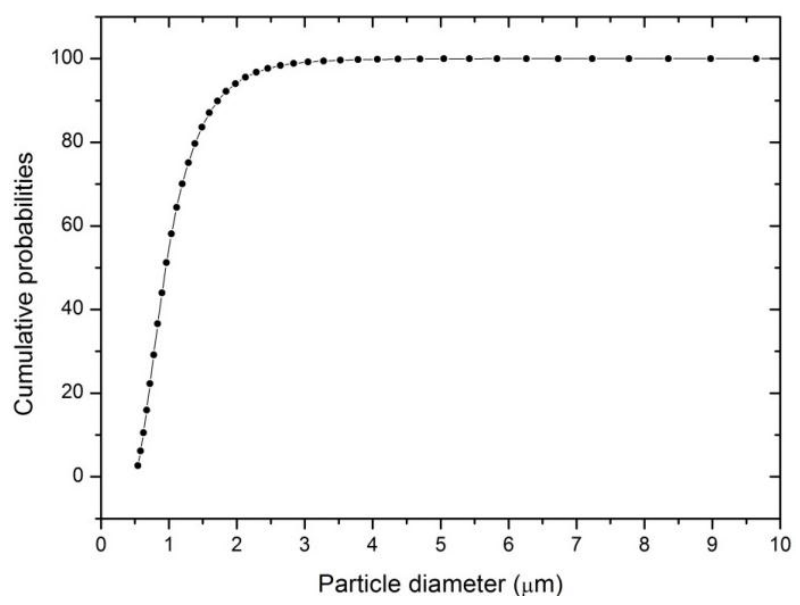

Figure 2 Geometry and sizes of plaster particles (a) geometry (b) sizes

\subsection{Experimental test rig}

The test rig consisted of a PVC pipe, a pitot tube, a particle feeder and a cyclonic 
vacuum cleaner, as shown in Figure 3. The air flow entered the vacuum cleaner through a $36 \mathrm{~mm}$ diameter and $1000 \mathrm{~mm}$ long PVC pipe. A Pitot tube, with a diameter of $1.2 \mathrm{~mm}$, was placed $65 \mathrm{~mm}$ behind the inlet of PVC pipe. The Pitot tube was connected to a pressure transducer (model Setra 239) with a range of $\pm 3736 \mathrm{~Pa}$ and an accuracy of $\pm 3 \mathrm{~Pa}$. The measured pressure data (with a sampling rate of $200 \mathrm{~Hz}$ ) were transmitted to a data acquisition card (model NI PCI-6221) and were stored in a PC computer. During the experiment, the mass flow rate of the test rig was fixed at 40 $\mathrm{g} / \mathrm{s} \pm 2 \mathrm{~g} / \mathrm{s}$. Particles were loaded at a rate of $5 \mathrm{~g} / \mathrm{min}$ for 20 minutes using a shaker (model V20/PA100 manufactured by Gearing \& Watson Ltd.) at a frequency of 100 Hz. In terms of mass ratio of particle to air, the particle load rate was $2 \mathrm{~g} / \mathrm{kg}$ in total. However, the actual particle load rate into the cyclone in the secondary separation stage was less than $2 \mathrm{~g} / \mathrm{kg}$, as some particles were captured in the primary separation stage. In this study the actual particle load rate into the cyclone was $1 \mathrm{~g} / \mathrm{kg}$ to $1.4 \mathrm{~g} / \mathrm{kg}$. 


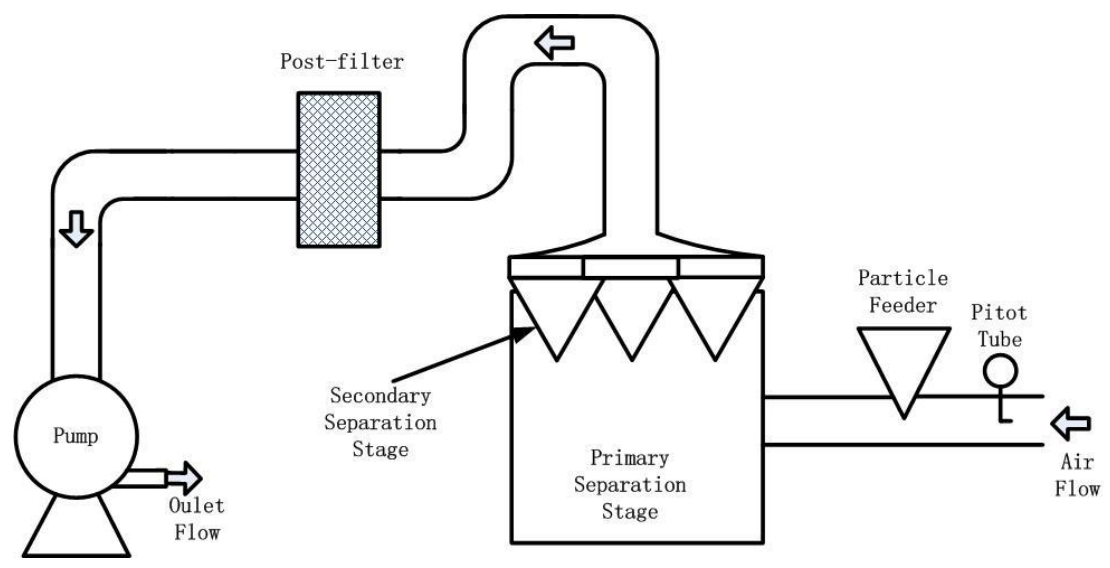

Figure 3 Illustration of the test rig

The vacuum cleaner consisted of two separation stages. The primary separation stage consisted of a cylindrical chamber and the secondary separation stage consisted of 14 cyclones, as shown in Figure 4. The flow passed through the primary separation stage first and then entered the secondary separation stage, where the 14 cyclones were in parallel, sharing the same inlet and outlet. Geometry of 14 cyclones was the same. Detailed dimensions as listed in Table 1. 
Table 1 Dimensions of the cyclone in the experiment

\begin{tabular}{cccc}
\hline Feature & Dimension, $\mathrm{mm}$ & Feature & Dimension, $\mathrm{mm}$ \\
\hline $\mathrm{a}$ & 5 & $\mathrm{~S}$ & 13.55 \\
$\mathrm{~b}$ & 11.6 & $\mathrm{Hc}$ & 12.1 \\
$\mathrm{D}$ & 35 & $\mathrm{~L}$ & 87.11 \\
$\mathrm{De}$ & 8.54 & $\mathrm{~d}$ & 6.6 \\
th & 1 & & \\
\hline \multicolumn{4}{c}{} \\
\end{tabular}

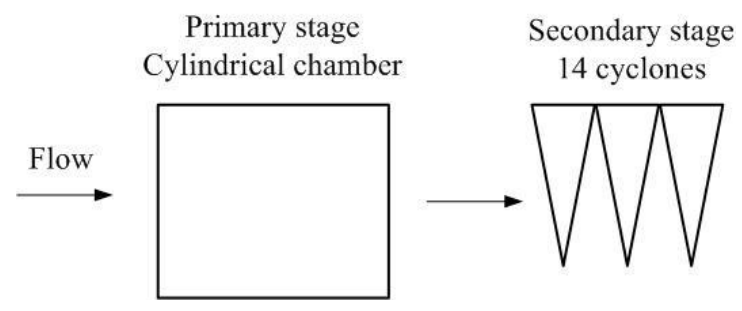

Figure 4 Illustration of two stages of vacuum cleaner

The air flow inlet condition of the primary separation stage was modified by placing a plastic wedge of $40 \mathrm{~mm} \times 40 \mathrm{~mm} \times 6 \mathrm{~mm}$ size at the inlet of the primary separation stage, as shown in Figure 5. 

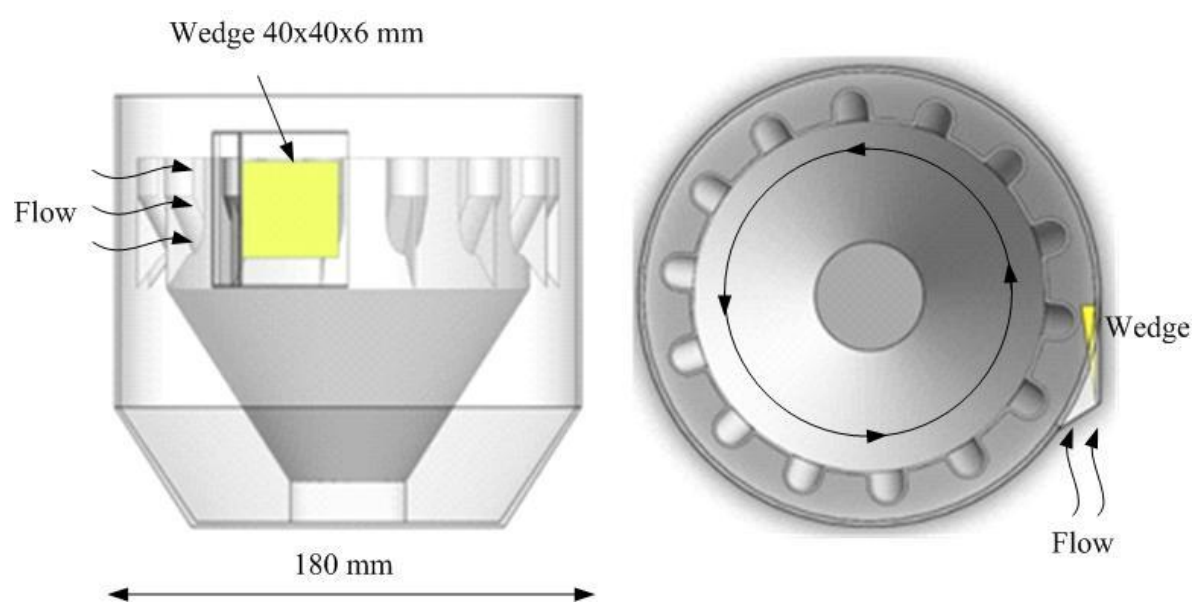

Figure 5 Sketch showing the location of the wedge at primary separation stage (side view and top view)

In the experiment, the particle adhesion was not even for the 14 cyclones. Some cyclones always had serious particle adhesion. The others had little particle adhesion. In order to minimize the measurement uncertainty of the particle adhesion, the cyclone with the highest amount of the particle adhesion was chosen for the experimental investigation. The location of the chosen cyclone relative to the primary separation stage is shown in Figure 6. 


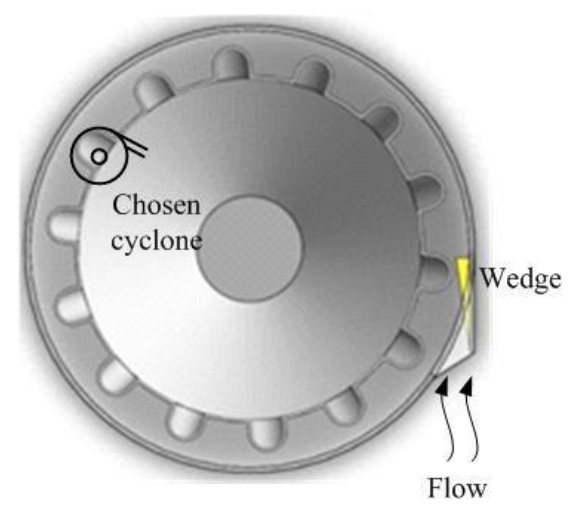

Figure 6 Location of the chosen cyclone

The environmental temperature and humidity during the experiment was $20 \pm 2^{\circ} \mathrm{C}$ and $50 \pm 5 \% \mathrm{RH}$, respectively. The variation of room pressure was less than $5 \%$ of $101 \mathrm{kPa}$ over one year, according to the data from centre of atmospheric science in the university. Thus, the test environment condition is regarded as constant.

\subsection{Experimental measurement techniques}

With the aid of the oil flow visualization technique, the flow direction on the wall of the primary separation stage was characterised discretely. The dye for the oil flow visualization was a mixture of the sunflower vegetable oil (53\% in weight), fluorescent powder (24\%) and the silicone oil with a viscosity of $5 \mathrm{~Pa}^{*} \mathrm{~s}(24 \%)$. Four lines of dye droplets (each line contains four dye droplets) were placed on an Acetate 
film of $490 \mathrm{~mm} \times 90 \mathrm{~mm} \times 0.1 \mathrm{~mm}$ in size that was glued onto the wall before the oil flow visualization test, as shown in Figure 7. After the oil flow visualization test, the Acetate film was taken off, so that the moving direction dye trajectory was measured.

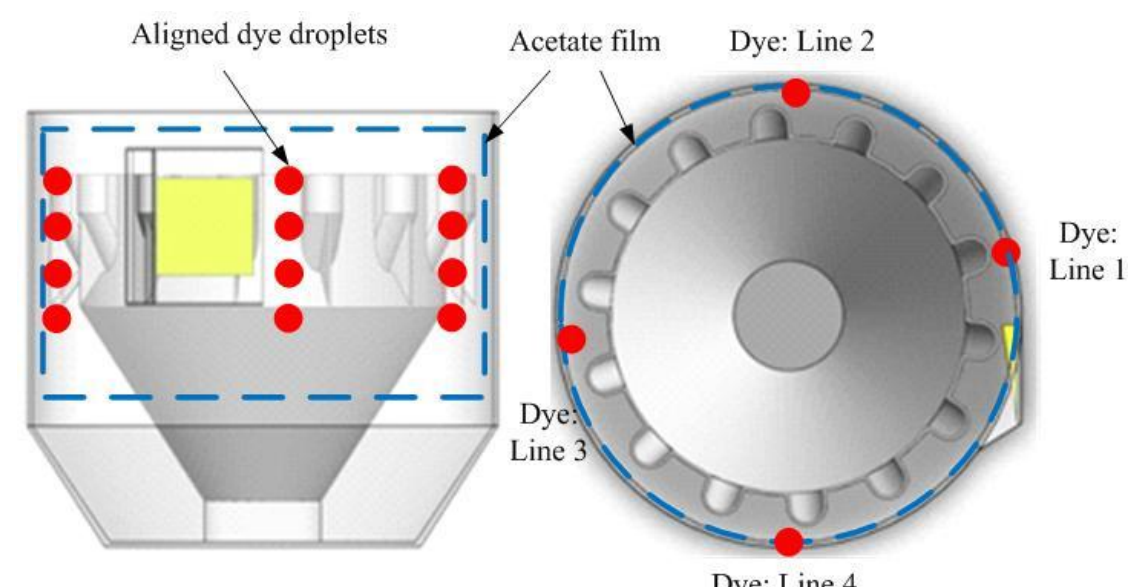

Figure 7 Location of dye droplets on Acetate film

The particle inlet position was determined by making use of the response time of a Pitot tube to a step change in the pressure. The Pitot tube had a diameter of $1.2 \mathrm{~mm}$ and it was placed parallel to the local flow direction at the cyclone inlet, as shown in Figure 8. The step signal in pressure was generated by stopping test for 1 minute followed by running test with particle loading for another minute. 


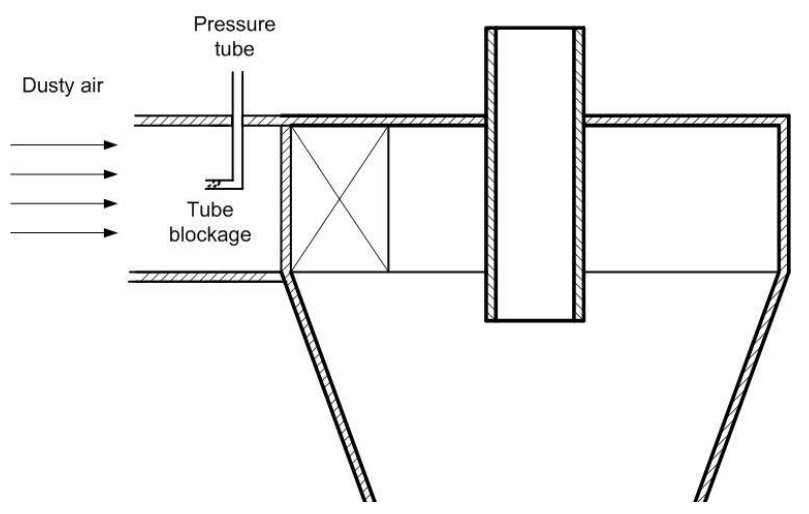

Figure 8 Illustration of particle inlet position measurement

In addition, the oil flow visualization technique was used to visualise the trajectory of flow motion at discrete location on the inner wall of the cyclone. Five holes, each with a diameter of $1.2 \mathrm{~mm}$, were drilled along the wall of the cyclone, as shown in Figure 9. The dye was injected into the cyclone through these holes under zero particle loading condition. Images of half cut cyclones with dye trajectories were then taken. The trajectory angle, defined as the complementary angle of the trajectory line with the axis of the cyclone, was measured with the aid of MATLAB programme. A change in the trajectory angle implied changes in the flow axial velocity and tangential velocity along the wall as the angle was proportional to the flow axial velocity to tangential velocity ratio. 


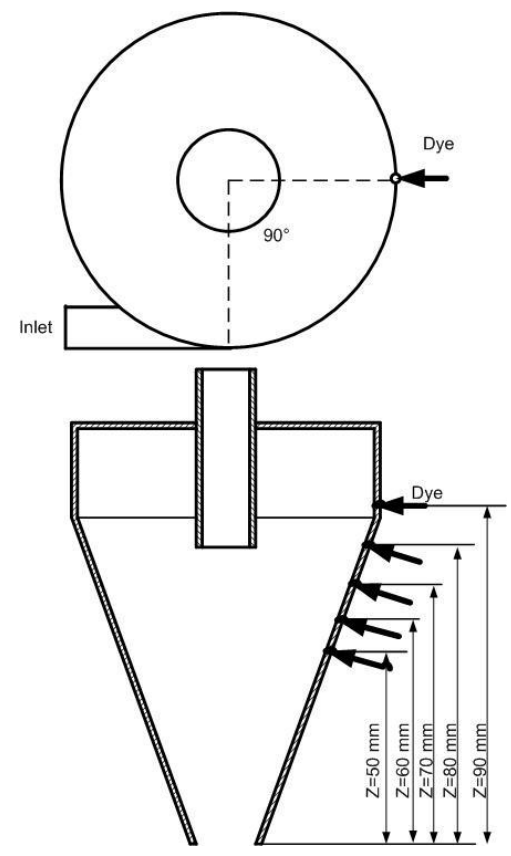

Figure 9 Location of dye injection holes in oil flow visualization experiment

The particle electrical static charges were measured using a digital coulomb meter (model XJC-205-010F), with a range of $\pm 1999 \mathrm{nC}$. The measurement was made by connecting the coulomb meter onto a wire that was connected to the inner surface of the cyclone wall coated with a thin layer of silver as shown in Figure 10. 


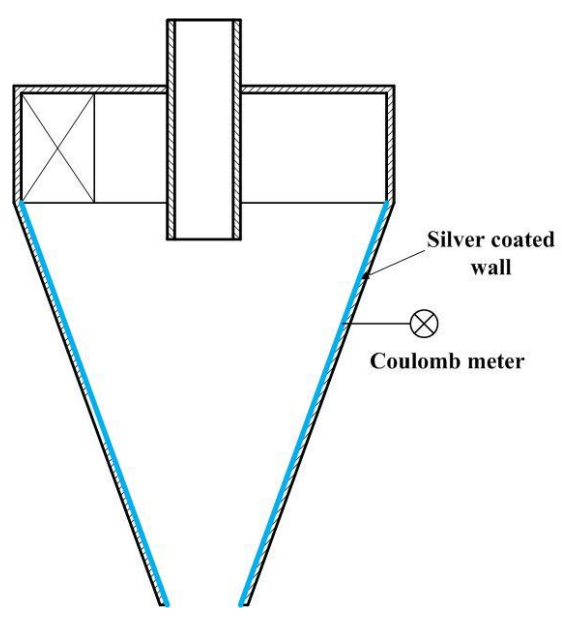

Figure 10 Illustration of particle charge measurement

The images of particle adhesion on the inner wall of the cyclone were taken with a Canon Pro1 CCD camera. Furthermore, those particles adhering onto the inner wall of cyclone were brushed off from the wall and were collected. Their weights were measured by a digital weight scale model Kern PCB 100-3 with a resolution of 0.001 g. The amount of particles entering the cyclone was taken as the average value of the amount of particles entering the secondary separation stage, which was deduced from the total amount of particles and the amount of particles collected in the primary separation stage.

\section{Results and Discussion}

In this study, it was noted that the weight of particles collected in the post-filter 
behind the secondary separation stage always ranged from $0 \mathrm{~g}$ to $1 \mathrm{~g}$. As the test duration was 20 minutes and the particle load rate was $5 \mathrm{~g} / \mathrm{s}$, the average separation efficiency of the cyclone in the secondary separation stage was higher than $99 \%$. Therefore, the amount of escaping particles was minor and it was neglected.

\subsection{Flow and particle trajectory in the primary separation stage}

As the wedge was placed at the inlet of the primary separation stage, it was believed that the flow and particle trajectory was modified. With the aid of the oil flow visualization on the wall of the primary separation stage, the flow direction was observed, as shown in Figure 11.

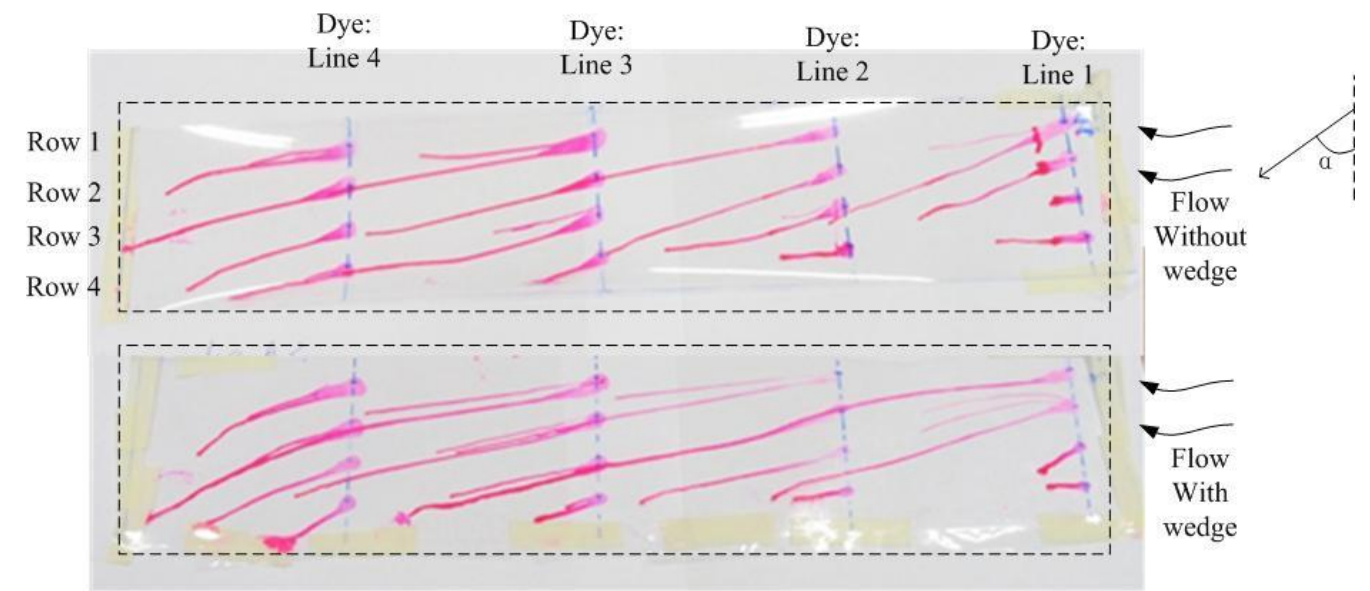

Figure 11 Oil dye trajectory on the wall of the primary separation stage

The angle of the flow direction, defined as the angle of $\alpha$, was measured. The oil 
droplet was labelled according to its location. For example, the droplet, located at the 'Dye: Line 2' and the 'Row 3', was labelled as the droplet '23'. As shown in Figure 12 , the majority of the flow direction was not changed more than 10 degrees, except the flow over droplets ' 11 ', ' 13 ', '24' and ' 44 '.

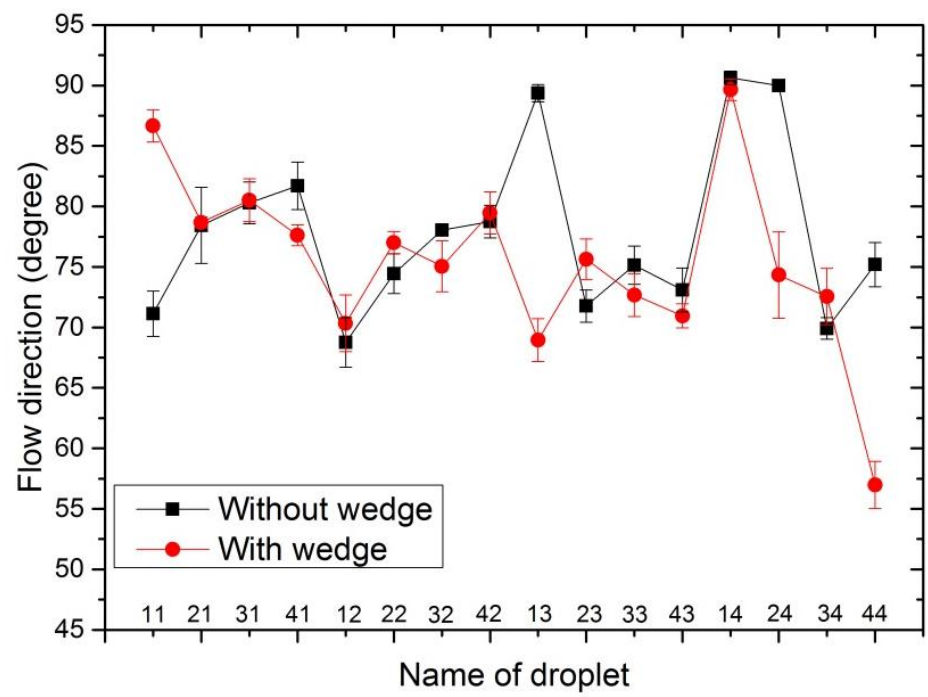

Figure 12 Angle of flow direction of the dye trajectory

By comparing the flow direction at ' 11 ' and ' 13 ', it was found that with the presence of the wedge, the flow over the dye droplet ' 11 ' raised up, and the flow over the dye droplet '13' turned down, as shown in Figure 13. For this reason, the flow passing the upper and lower edge of the wedge was diverted, as illustrated in Figure 14. 

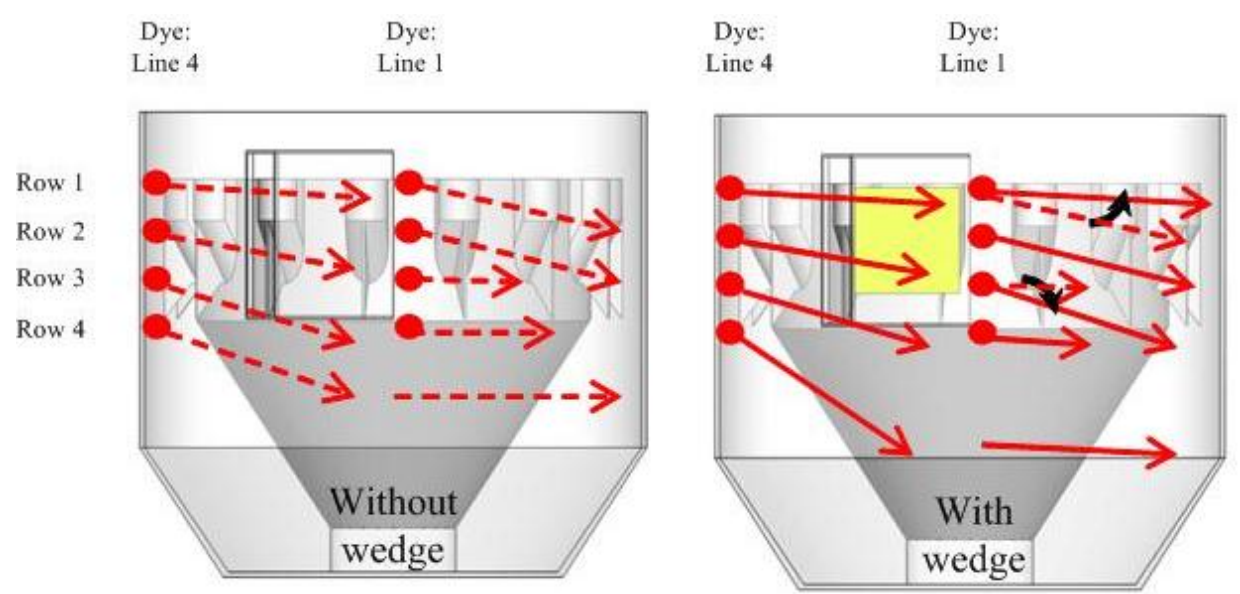

Figure 13 Sketch showing the flow on the wall of primary separation stage

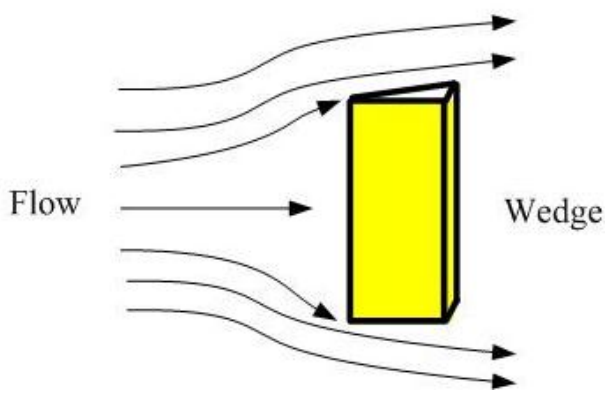

Figure 14 Sketch showing the flow passing the wedge

From the above analysis, the change of the flow direction in the primary separation stage was believed to influence the particle trajectory. Therefore, images of particle trajectory in the primary separation stage were taken. The white particle trajectory was found to be lower without the wedge compared with that with the wedge, as shown in Figure 15. 

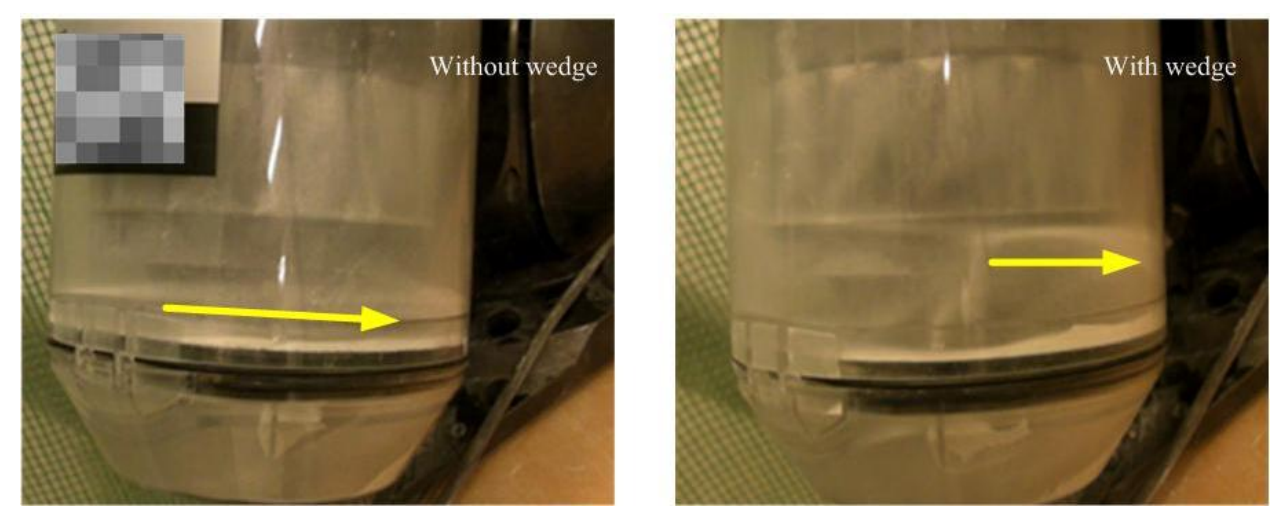

Figure 15 Particle trajectory in the primary separation stage

\subsection{Effect of the wedge on the particle adhesion in the cyclone}

Without the wedge, the particle adhesion in spiral patterns (SPs) on the wall of the chosen cyclone was observed with a thicker adhesion layer (TAL) at the cyclone tip. In contrast, such particle adhesion patterns were not clearly observed with the wedge, as shown in Figure 16. 
$\mathbf{a}$

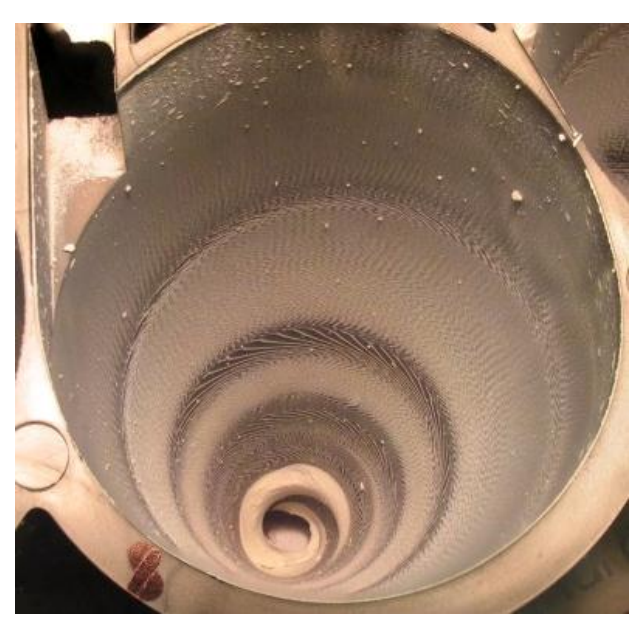

b

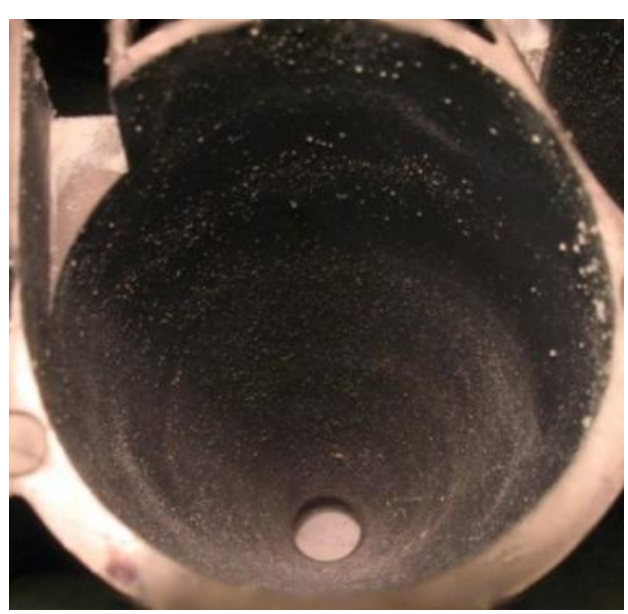

Figure 16 Particle adhesion patterns in the cyclone (a) without wedge (b) with wedge

The weight of the particle adhesion decreased from $0.16 \pm 0.021 \mathrm{~g}$ to $0.01 \pm 0.003 \mathrm{~g}$ with the presence of the wedge, corresponding to a reduction of $94 \%$. On the other hand, the weight of particles entering the cyclone (using average results of the secondary separation stage) was found to slightly increase from $3.13 \pm 0.537 \mathrm{~g}$ to 4.55 $\pm 0.497 \mathrm{~g}$ (45\% increment) with the wedge. Hence, the particle adhesion in the cyclone was reduced with the wedge even though the weight of particles entering the cyclone had increased slightly.

As experimental results of the weight of particles into the chosen cyclone were average results of 14 cyclones, it is better to have a look at the overall particle adhesion of the 14 cyclones. The overall weight of the particle adhesion in 14 
cyclones is $1.76 \pm 0.113 \mathrm{~g}$ without wedge, compared with that of $0.72 \pm 0.078 \mathrm{~g}$ with wedge. Therefore, the wedge reduced about $60 \%$ of the overall particle adhesion in 14 cyclones. This finding confirmed the effect of the wedge on the reduction of the particle adhesion.

\subsection{Possible factors accounting for the effect of the wedge}

\subsubsection{Effect of the wedge on the flow direction and mass flow rate}

Since the wedge changed the flow direction in the primary separation stage, the flow direction in the cyclone might be changed, which can be indicated by the angle of the dye trajectory in the cyclone. The cyclone was half cut to enable the measurement of flow direction in it, as shown in Figure 17.

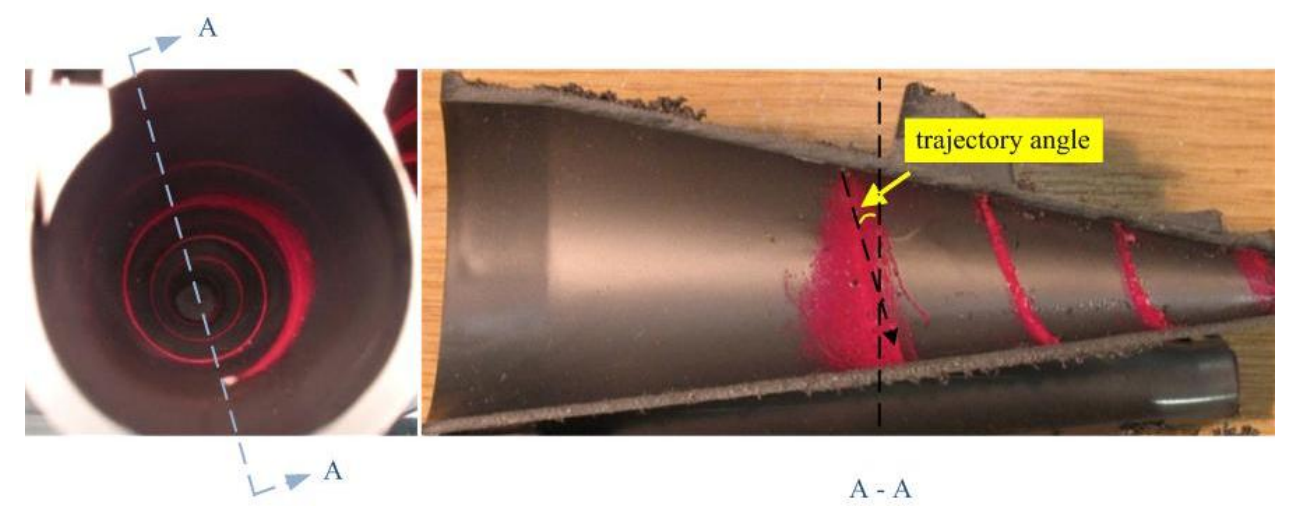

Figure 17 Half cut cyclone for the flow direction measurement 
The measured results are shown in Figure 18. It can be seen that the difference of the flow direction for both conditions was less than 5 degrees. Since the difference of the flow direction was within the measurement uncertainty (up to 10 degrees), it was expected that the flow direction in the cyclone remained essentially for both conditions.

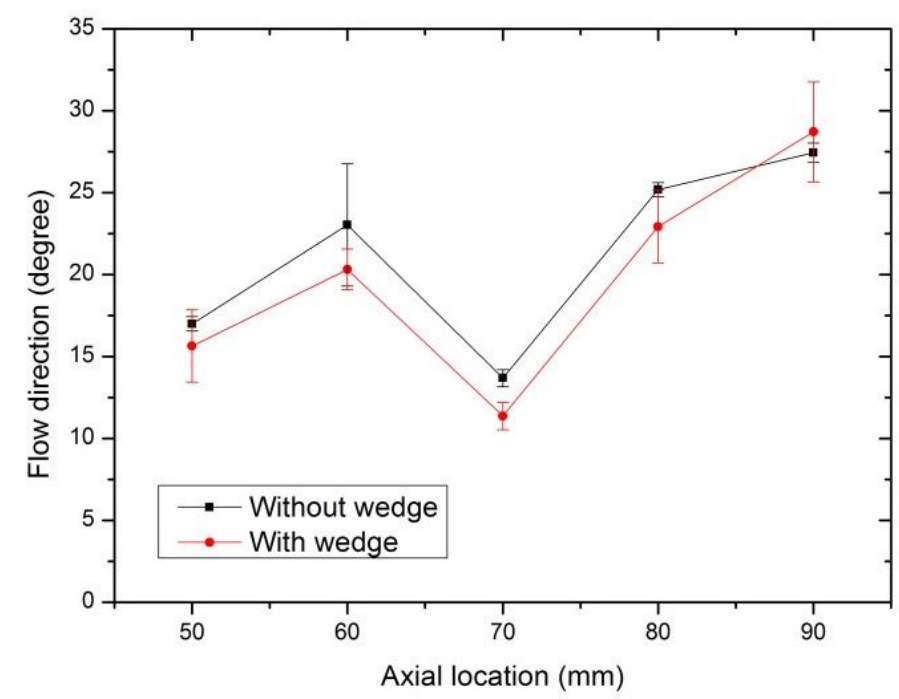

Figure 18 Effect of wedge on the flow direction in the cyclone

As it was not possible to measure the mass flow rate of the cyclone, the mass flow rate of the vacuum cleaner was measured for both conditions when the particles were not loaded. Results are shown Figure 19. It can be seen that there was no difference of the mass flow rate between the two conditions. Therefore, the mass flow rate of the 
cyclone was regarded as not being affected by the wedge.

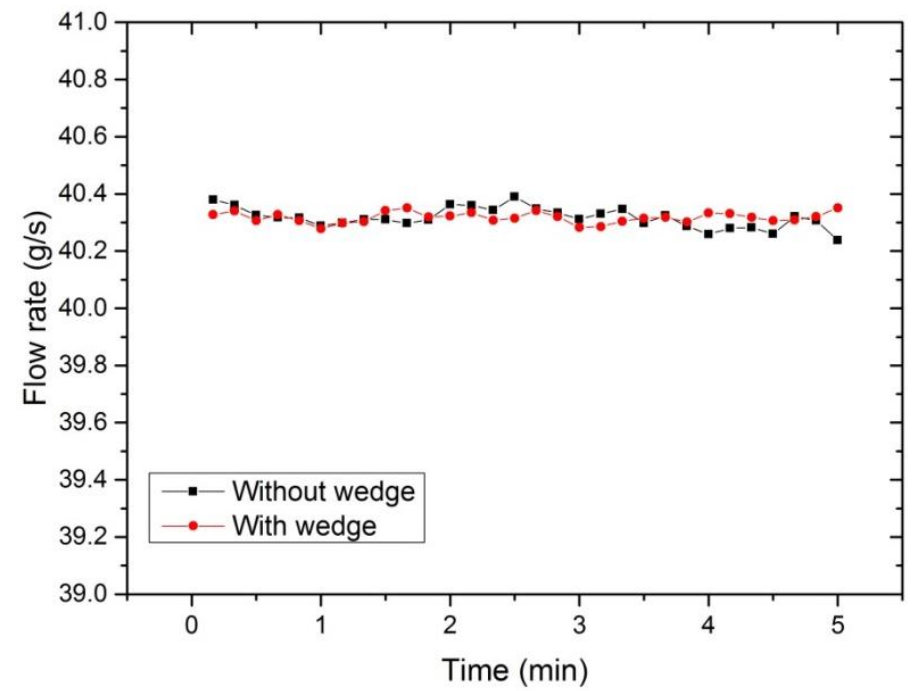

Figure 19 Effect of wedge on the mass flow rate of the vacuum cleaner

\subsubsection{Effect of the wedge on the particle inlet position}

As the particles inlet position of the cyclone was affected by the particle trajectory in the primary separation stage, the particle inlet position of the cyclone was measured. The pressure tube was placed at four different locations in the inlet cross section of the cyclone as shown in Figure 20. The four locations were labelled as 'I', 'II', 'III' and 'IV', respectively. 


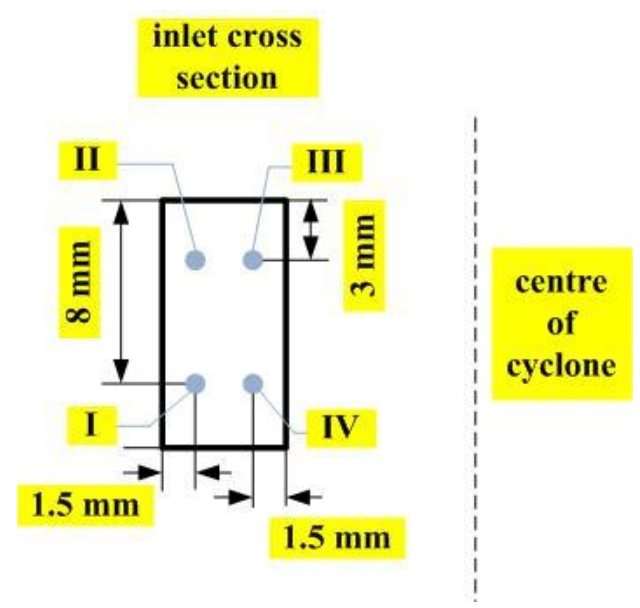

Figure 20 Locations of the pressure tube in the inlet cross section of the cyclone

Five step signals were generated during the test. An example of the measured pressure is shown in Figure 21. It can be seen that the measured pressure had a fast dynamic response at the beginning that had a square profile. As the time increased and more particles were added, the dynamic response of the pressure tube decreased, resulting in a curved profile. Therefore, when the pressure tube was blocked by the particles, its dynamic response time was deteriorated. It can be expected that the slowest dynamic response time was corresponded to the highest particle concentration. 


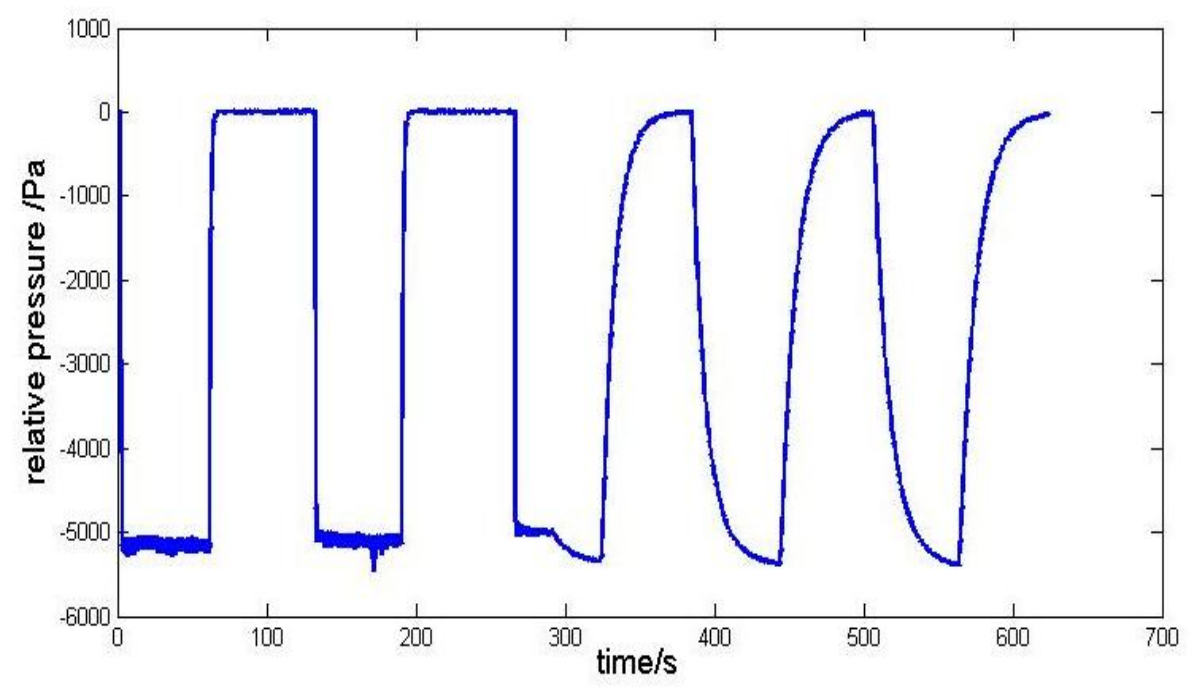

Figure 21 An example of the pressure of five step signals

The average dynamic response time of five step signals was calculated. As shown in Figure 22, the average dynamic response time was the longest at the location 'I' and shortest at the location 'II' without the wedge, indicating a high particle concentration at the lower left of the inlet. On the contrary, the average dynamic response time was the longest at the location 'II' and shortest at the location 'I' with the wedge, indicating a high particle concentration in the upper left of the inlet. Therefore, the particle inlet position at the inlet had been changed by the wedge. The aforementioned change in the particle inlet position of the cyclone was associated with the change of the particle trajectory in the primary separation stage. 


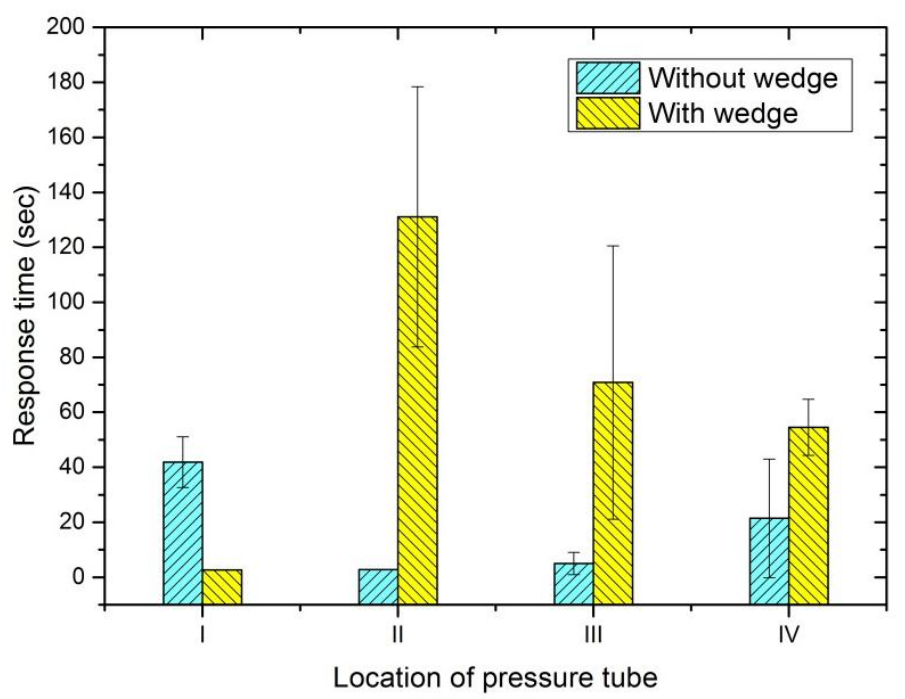

Figure 22 Average dynamic response time of the pressure tube at different locations with and without the wedge

\subsubsection{Effect of the wedge on the particle size and particle charge}

The particles size inside the cyclone at both conditions was measured by TSI 3321. As shown in Figure 23, the distribution of the particle size at both conditions appeared to be very similar. In addition, the particle charge was measured by the digital coulomb meter. As shown in Figure 24, the cumulative particle charge at both conditions was similar. The cumulative particle charge increased linearly during the first 5 minutes at a rate of $420 \mathrm{nC} / \mathrm{min}$ before it reached the measurement limitation of the digital coulomb meter (1999 nC). 


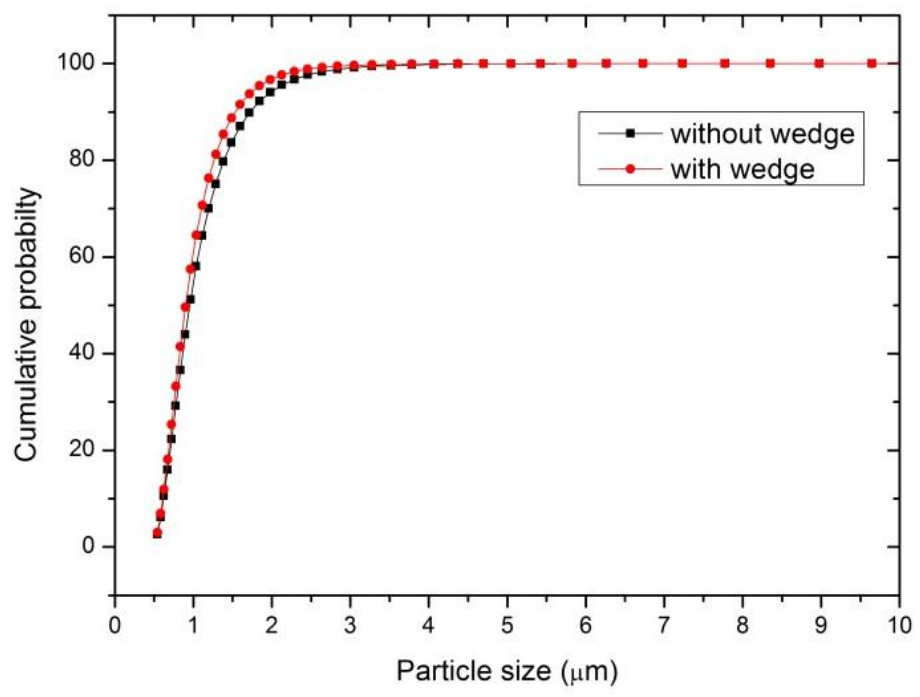

Figure 23 Effect of wedge on the particle size

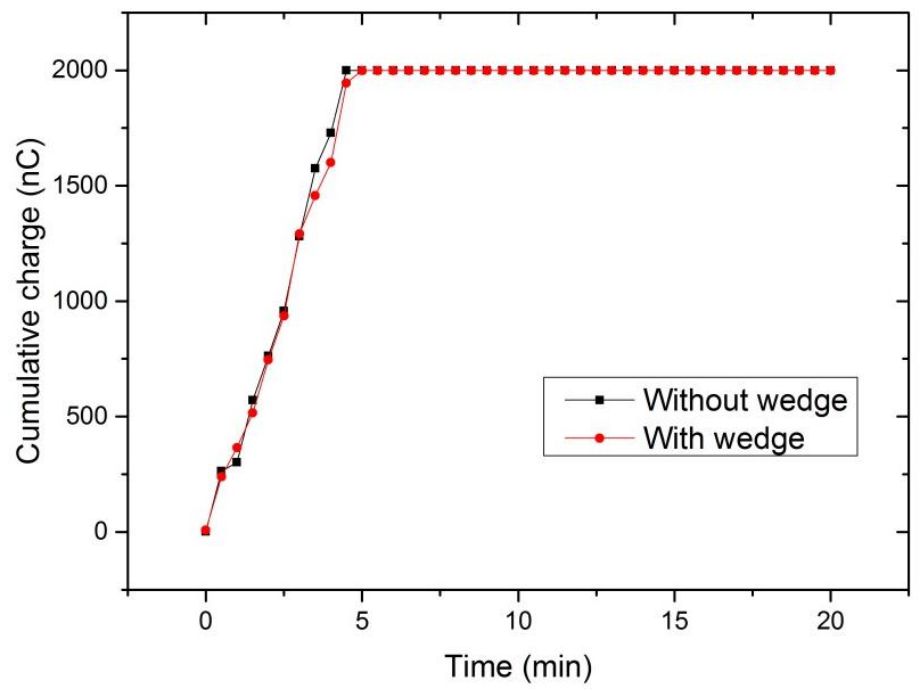

Figure 24 Effect of wedge on the particle charge 


\subsection{Discussion}

A wedge placed in the primary separation stage was found to divert the flow behind it, which affected the particle trajectory in the primary separation stage, resulting in the change of the particle inlet position in the chosen cyclone. It was found that the particle adhesion in the cyclone was reduced about $94 \%$ by the wedge, even the weight of the particles into the cyclone was increased by $45 \%$.

Since the environment condition, Hamaker constant, particle charge and the particle size in the cyclone were not changed by the wedge, the capillary forces, van der Waals forces and the electrostatic forces remained the same for the with wedge condition and without wedge condition. Therefore, only the aerodynamic forces were affected by the wedge. Furthermore, as the particle size was not changed, the aerodynamic forces were determined by the flow velocity only.

In our experiment, it was found that the flow direction and velocity (without particles being loaded) did not vary with the wedge. However, the flow direction and velocity can be changed by loading the particles (Chu et al., 2011; Chan et al., 2008), due to the two way coupled particle-flow interaction. As we found that the particle inlet position was changed by the wedge, it was believed that different particle inlet 
positions would lead to different particle-flow interactions. Therefore, the aerodynamic forces were changed by the wedge, which was the reason for the reduction of the particle adhesion.

Wang et al. (2006) found that the separation efficiency was different with different particle inlet position. However, in our experiments the separation efficiency was not significantly changed by the wedge, i.e. the particle inlet position, because the separation efficiency was always higher than $99 \%$. It might be because that the cyclone used in our experiment was 5 times smaller than that in the previous study (Wang et al., 2006).

\section{Conclusion}

In this study, an easy way to reduction the particle adhesion in the cyclone was developed by placing a wedge $(40 \times 40 \times 6 \mathrm{~mm})$ at the inlet of the primary separation stage. It was found that the wedge can reduce the particle adhesion in the cyclone by $94 \%$ at a particle load rate of $5 \mathrm{~g} / \mathrm{min}$ and test duration of 20 minutes. The factor contributing to the reduction of the particle adhesion was due to the change of the particle inlet position. Without the wedge, the major particle inlet position was at the lower left part of the inlet. With the wedge, the major particle inlet position was 
changed to the upper left part of the inlet.

By comparing the factors that may affect the particle adhesion in the cyclone, it was believed that the change of the particle inlet position would lead to different particle-flow interactions in the cyclone, resulting in different aerodynamic forces on the particles.

\section{Future Work}

In this study, due to the limitation of experimental test rig (vacuum cleaner based test rig), the study was focused on finding out the reason for particle adhesion reduction with wedge. Future work is required on the optimization of shape and position of wedge. Furthermore, the operation condition of the cyclone was fixed at a single point due to the nature of the vacuum cleaner. It requires future work to investigate the effect of mass flow rate, particle load rate, particle material property, and cyclone geometry with and without wedge.

\section{Acknowledgement}

The author would like to acknowledge the Dyson Ltd. for funding this research. Also thanks for James Allan in National Centre for Atmospheric Science (NCAS) for the 
assistance with particle size measurements.

\section{References}

Chang, Y.F., Ilea, C.G., Aasen, Ø.L. and Hoffmann, A.C. (2011). Particle flow in a hydrocyclone investigated by positron emission particle tracking. Chemical Engineering Science, 66(18), pp.4203-4211.

Chu, K.W., Wang, B., Xu, D.L., Chen, Y.X. and Yu, A.B. (2011). CFD-DEM simulation of the gas-solid flow in a cyclone separator. Chemical Engineering Science, 66(5), pp.834-847.

He, S., Zeng, J. and Li, S. (2014) High-temperature cyclone separator anti-blocking apparatus and method. Patent CN104147858A

Hoffmann, A. C., and Stein, L. E. (2002). Gas cyclone and swirl tubes. Springer-Verlag Berlin Heidelberg.

Houben, J. J. H. (2011). Experimental investigations and CFD simulations on particle depositions in gas cyclone. Ph. D. thesis, Montanuniversitaet Leoben.

Huang, M., Zhou, P. and Yang, J. (2013) Anti-blockage device of cyclone 
separator. Patent CN203454113U

Mittal, K. L., \& Jaiswal, R. (2015). Particle Adhesion and Removal. John Wiley \& Sons.

Mozley, R. H. (1979). Cyclone separator. Patent US4148723

O'Callaghan, D., \& Cunningham, P. (2005). Modern process control techniques in the production of dried milk products-a review. Le Lait, 85(4-5), 335-342.

Room pressure data: http://www.cas.manchester.ac.uk/restools/whitworth/plots/ (accessed on $30^{\text {th }}$ March, 2016)

Wang, B., Xu, D.L., Chu, K.W. and Yu, A.B. (2006). Numerical study of gas-solid flow in a cyclone separator. Applied Mathematical Modelling, 30(11), pp.1326-1342. 